\title{
La adquisición del léxico veterinario (alemán- español): una propuesta basada en corpus para la traducción de textos
}

\author{
María del Carmen Balbuena Torezano \\ Universidad de Córdoba \\ mcbalbuena@uco.es \\ https://dx.doi.org.10.12795/futhark.2019.il4.02
}

Fecha de recepción: 15.05.2018

Fecha de aceptación: 1.07.2019

Resumen: La profesión veterinaria abarca un elevado número de campos y disciplinas y ámbitos de actuación, entre las que caben señalar la medicina veterinaria, el control de la calidad alimentaria, la salud pública y laboral, la producción animal, el bienestar animal o la industria farmacéutica, entre otros. Ello supone que los textos sobre los que se sustentan estas especialidades contienen una alta densidad léxica, como sucede con otros campos de las Ciencias de la Salud. En este trabajo ofrecemos una propuesta para la adquisición y el aprendizaje del léxico especializado del sector veterinario en el par de lenguas alemán-español.

Palabras clave: traducción especializada; competencia léxica; textos veterinarios; adquisición del léxico; lengua alemana.

\section{Lexical acquisition of veterinary lexicon (German-Spanish): a proposal based on corpus for the translation}

\begin{abstract}
The veterinary profession covers a huge variety of fields, disciplines and areas of interest, including veterinary medicine, food quality control, public and occupational health, animal production, animal welfare or pharmaceutical industry, among others. This means that the texts on which these disciplines are based contain a high lexical density, as is the case with other fields in Health Sciences. In this paper we focus on a proposal for lexical acquisition and learning of specialized terminology for veterinary contexts for the combination German-Spanish.
\end{abstract}

Keywords: Specialized translation; lexical translation competence; veterinary texts; lexical acquisition; German language. 
Sumario: Introducción. I. Competencia léxica y traducción. 2. Corpus y traducción. 3. Propuesta para la adquisición del léxico veterinario basado en corpus. 3.I. Creación del corpus textual. 3.2. Análisis del corpus con Sketch Engine. 3.2.I. Selección de términos. 3.2.2. Estudio de las concordancias. 4. Resultados y discusión. Conclusiones.

\section{Introducción}

La traducción de textos veterinarios, en tanto que traducción especializada, entraña diversas dificultades, entre la que se encuentra el conocimiento de las unidades léxicas y fraseológicas especializadas de este sector. En efecto, tener la competencia léxica, es decir, conocer los términos, supone (Jiménez 2002: 155): a) conocer su gramática, su pronunciación y su ortografía; b) conocer su morfología; c) conocer su colocabilidad; d) conocer sus restricciones sintácticas; d) conocer sus restricciones sintácticas; e) conocer su frecuencia en lengua oral y lengua escrita; f) conocer en qué contextos se puede utilizar; g) conocer sus relaciones semánticas y sintácticas con otras palabras; $h$ ) reconocer la palabra en su forma oral o escrita; i) recuperar la palabra cuando se necesita; j) conocer su significado conceptual y referencial; k) conocer los sentidos que connota y l) conocer el sentido pragmático. En el caso de la traducción, hemos de exigir esta competencia léxica en ambas lenguas de trabajo: la lengua original y la lengua meta. En las páginas siguientes ofreceremos una propuesta para la adquisición de la competencia léxica, en tanto que subcompetencia translativa, dentro del sector veterinario, y para el par de lenguas alemán-español.

\section{Competencia léxica y traducción}

La competencia léxica, afirma Jiménez (2002: 152), es “el conocimiento que se debe poseer para poder utilizar la palabra con propiedad (...) la capacidad de reconocer, aprender, recuperar y relacionar las distintas palabras a nivel oral y escrito". Esta cuestión, ampliamente debatida por los especialistas en el ámbito de la Lingüística Aplicada, es también abordada en el seno de la Traductología (Newmark 1988; Baker 1992, Delisle 1993; Sánchez 2013 y 2017).

Tal es la relevancia de la competencia léxica, que autores como Sánchez (2013) determinan que

(...) cuanto mayor sea el conocimiento léxico en las distintas lenguas de trabajo, mayor será la rapidez y calidad del trasvase y más rentable resultará el desempeño de la profesión. Así, es conveniente reflexionar sobre la necesidads (y la forma) de rescatar el componente léxico para mejorar la formación del traductor y del intérprete. 
Es decir, que la adquisición del léxico adecuado al ámbito de la traducción en la cual se enmarca el texto al que nos enfrentamos, supondrá una mayor posibilidad de llevar a buen término la traducción que tenemos ante nosotros, por un lado; por otro, garantizará que el TM resultante tiene la calidad necesaria que se espera de una traducción profesional. En este sentido, resulta de singular relevancia no sólo el dominio de las distintas técnicas y estrategias de traducción o la capacidad de resolución de problemas, sino también el conocimiento del léxico especializado que forma parte del TO.

\section{Corpus y traducción}

El uso de corpora textuales ad hoc para el estudio de la terminología de determinados ámbitos resulta de especial utilidad a la hora de analizar y seleccionar los candidatos a término. En este sentido, seguimos los parámetros aplicados por López y Olmo (2017) para la confección de un corpus textual alemán relativo al ámbito de la bioquímica, con un elevado nivel de especialización. Para ello, se toman como referencia criterios externos, tales como el contexto sociocultural y la función comunicativa, o la cantidad y la calidad de los textos seleccionados; por otra parte, criterios internos, relativos a aspectos únicamente lingüísticos, tales como la distribución de los términos, los aspectos lexicológicos y gramaticales, etc., también resultan de relevancia a la hora de elegir o desechar un texto determinado para la confección final del corpus.

\section{Propuesta para la adquisición del léxico veterinario basado en corpus}

\section{I. Creación del corpus textual}

Para desarrollar la propuesta que aquí ofrecemos hemos creado un corpus ad hoc compuesto por una treintena de textos, entre los que caben señalar un acuerdo internacional; tres decisiones de ejecución; dos directivas; diez reglamentos; y quince sentencias. Los textos han sido recuperados de EurLex (https://eur-lex.europa.eu/homepage.html).

\subsection{Análisis del corpus con Sketch Engine}

Una vez seleccionado el corpus textual sobre el que deseamos realizar el estudio, empleamos como herramienta de análisis Sketch Engine', software que analiza el comportamiento de las lenguas, bien de forma monolingüe, bien en

I En adelante: SE. 
comparación con otras. Dadas las posibilidades que ofrece SE, el primero de los pasos es seleccionar aquellos candidatos a término que puedan resultarnos de utilidad en este trabajo. Para ello, seleccionamos la opción de análisis de frecuencia dentro del corpus textual que hemos confeccionado. Una vez determinados estos términos, emplearemos la función colocacional que nos ofrece el software, de forma que seamos capaces de visualizar todas las posibles colocaciones de los términos elegidos para su estudio.

\subsection{Selección de términos}

Una vez configurado el corpus textual, es posible determinar aquellos términos que aparecen con mayor frecuencia en ellos. De este modo, obtenemos la siguiente pantalla, que nos ofrece el número de apariciones de cada término del corpus.

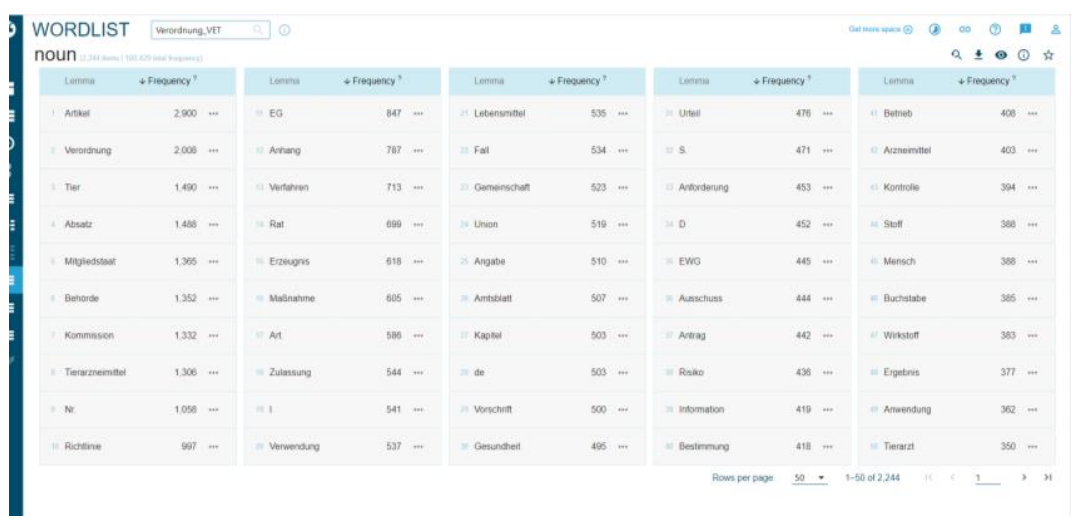

Figura I: Listado de frecuencias de los términos con SE

\subsubsection{Estudio de las concordancias}

Una vez seleccionado los términos más frecuentes, podemos estudiar las concordancias de cada uno de ellos, haciendo uso de una de las opciones que nos ofrece SE. Así, por ejemplo, podemos ver las construcciones en las que un término aparece en nominativo, acusativo, dativo o genitivo; las construcciones junto a verbos, preposiciones y grupos preposicionales; los posibles modificadores de dicho término; o su función como complemento de acusativo, dativo o genitivo, o bien los complementos que le acompañan. Tomemos, por ejemplo, el término Zoonoseerreger. Tras una búsqueda de este sustantivo con SE, y dentro del corpus que hemos confeccionado, obtenemos las siguientes colocaciones y parámetros gramaticales: 
a) Construcciones en dativo:

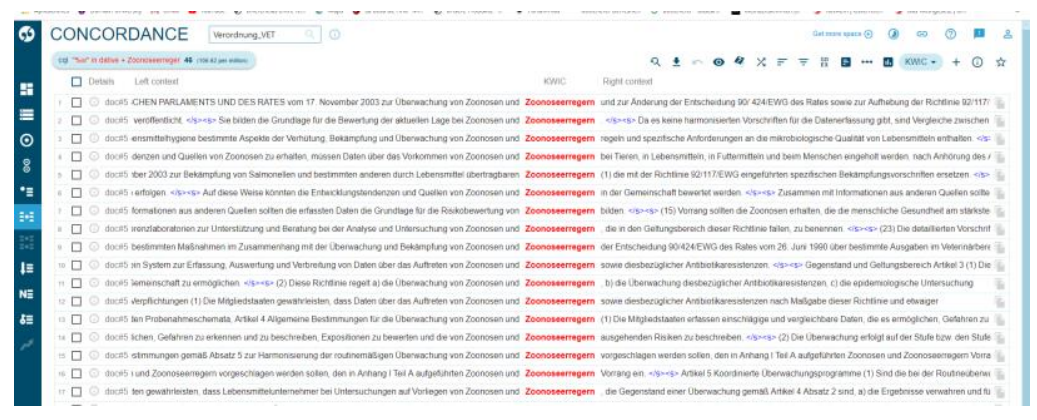

b) Construcciones en acusativo:

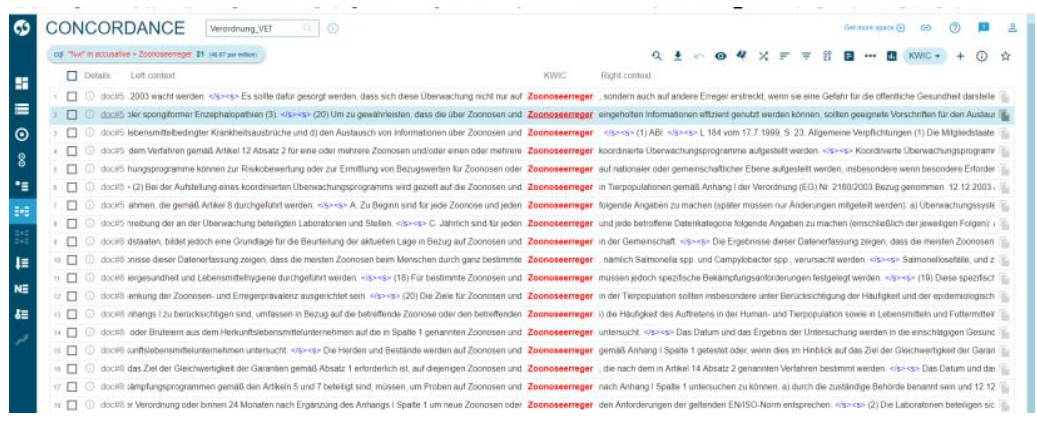

c) Construcciones en genitivo:

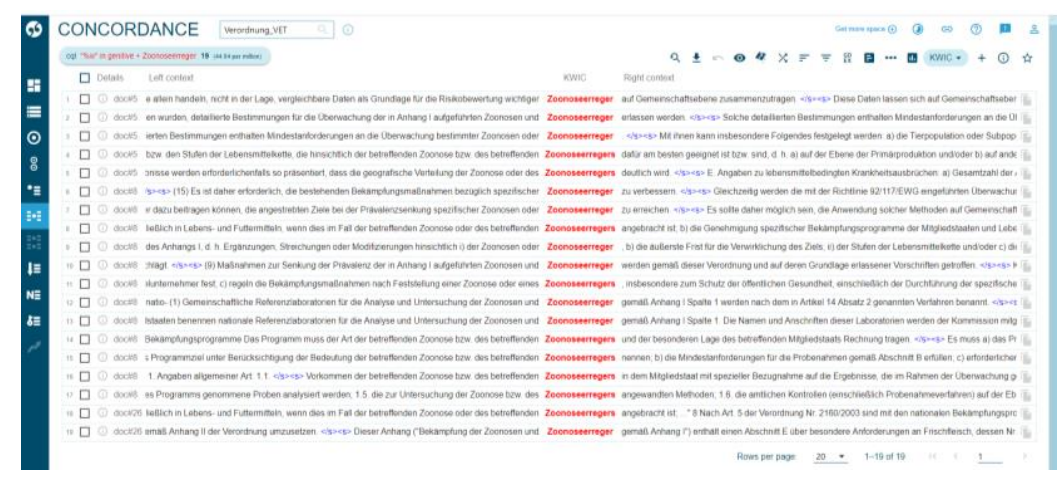




\section{d) Construcciones en nominativo:}

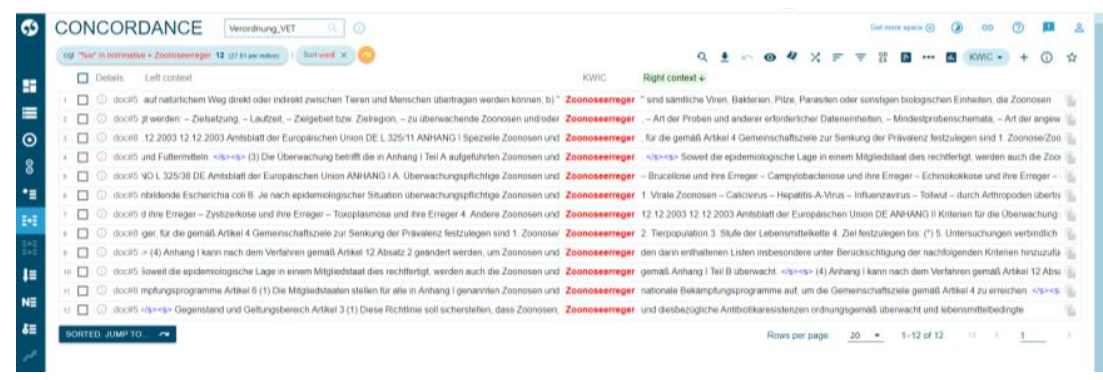

\section{e) Modificadores:}

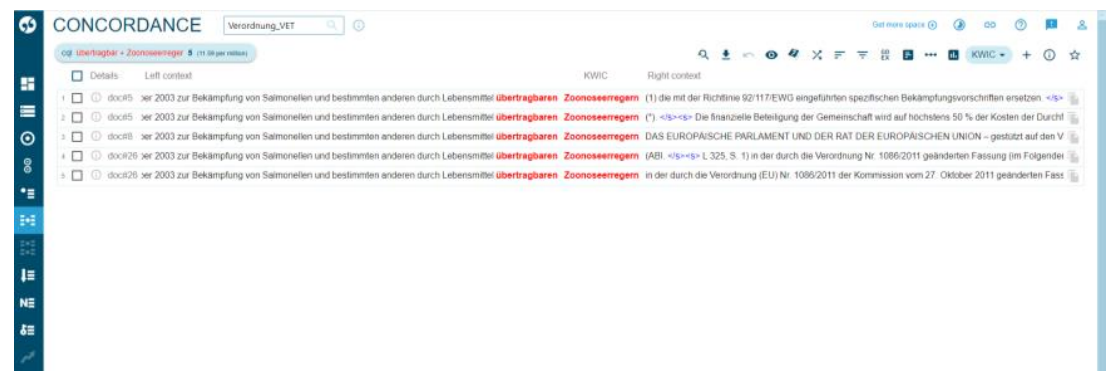

f) Complementos de genitivo de Zoonoseerreger:

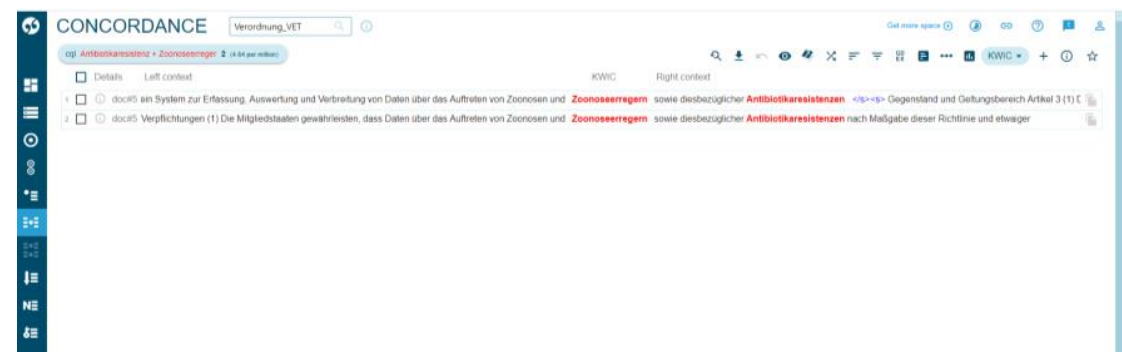


g) Complementos de dativo de Zoonoseerreger:

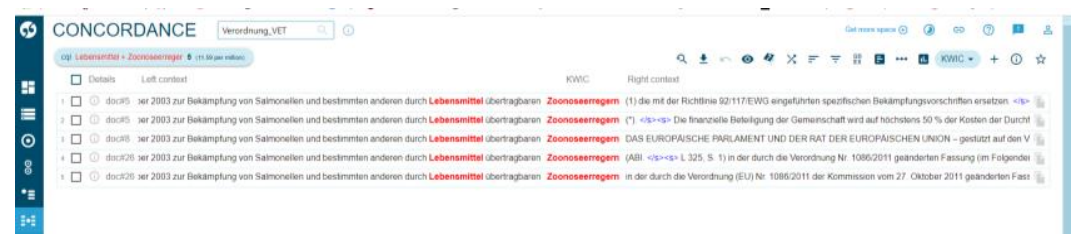

h) Zoonoseerreger como complemento en acusativo:

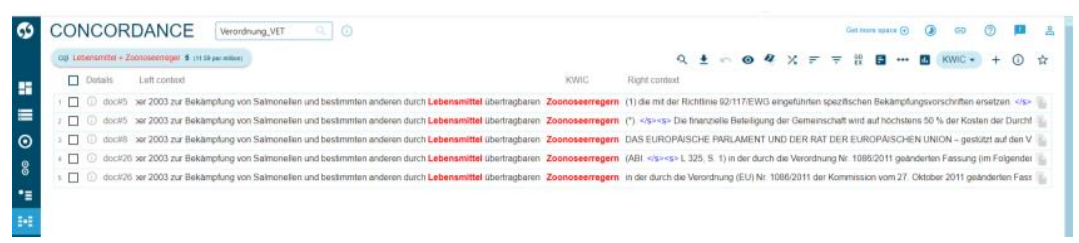

i) Verbos con Zoonoseerreger como complemento genitivo:

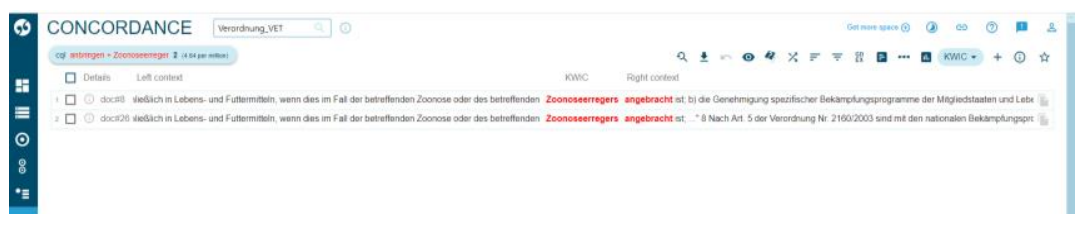

j) Verbos con Zoonoseerreger como complemento dativo: 
k) Verbos con Zoonoseerreger como complemento acusativo:

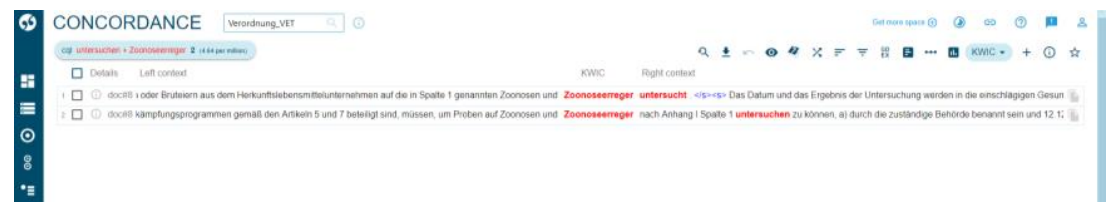

l) Construcciones con conjunciones copulativas (und/oder):

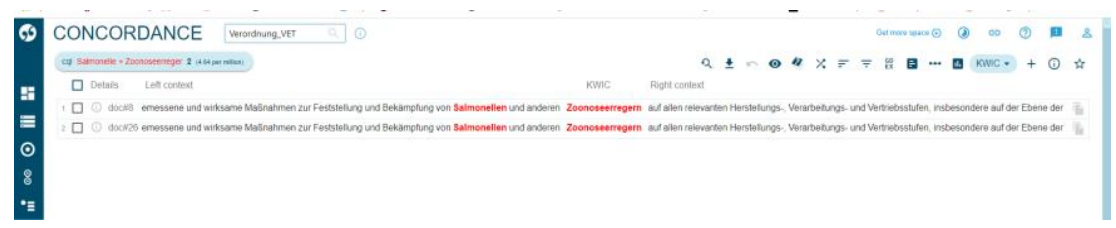

m) Preposiciones con Zoonoseerreger como objeto:

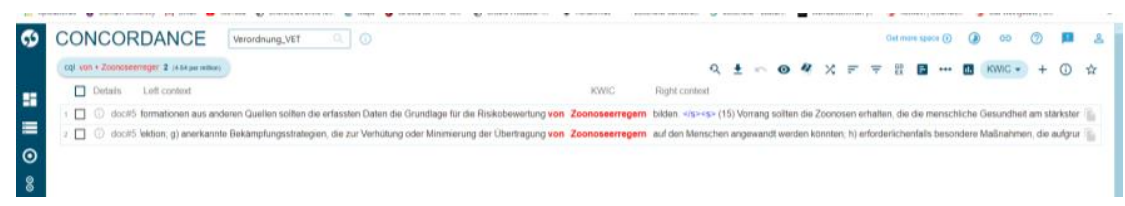

n) Construcciones con preposición:

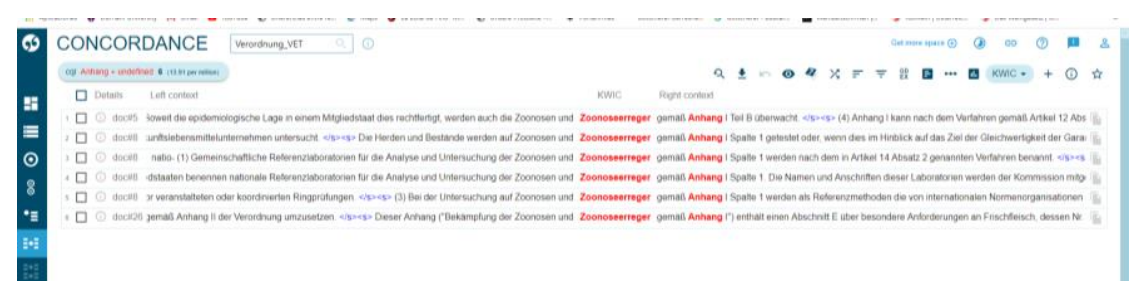

\section{Resultados y discusión}

Del estudio efectuado sobre el corpus textual a través de SE resultan un total de 26 términos del dominio léxico [veterinaria] que tienen una frecuencia de aparición susceptible de ser considerada lo suficientemente representativa para el estudio. Son los siguientes: 


\begin{tabular}{|l|l|}
\hline Tier & 1490 \\
\hline Tierarzneimittel & 1306 \\
\hline Maßnahme & 605 \\
\hline Lebensmittel & 535 \\
\hline Gesundheit & 495 \\
\hline Risiko & 436 \\
\hline Arzneimittel & 403 \\
\hline Kontrolle & 394 \\
\hline Stoff & 388 \\
\hline Mensch & 388 \\
\hline Wirkstoff & 383 \\
\hline Tierarzt & 350 \\
\hline Inverkehrlungen & 268 \\
\hline Lebensmittelunternehmer & 264 \\
\hline Fleisch & 264 \\
\hline Pfizer & 262 \\
\hline Futtermittel & 262 \\
\hline Herstellung & 260 \\
\hline Nebenprodukt & 241 \\
\hline Person & 222 \\
\hline Zusatstoff & 206 \\
\hline Virgimanycin & 202 \\
\hline Schutz & 197 \\
\hline Arzneibuch & 171 \\
\hline Rind & 164 \\
\hline Antibiotikum & 160 \\
\hline & \\
\hline & 260 \\
\hline
\end{tabular}


Veamos algunos ejemplos de concordancias a partir del corpus seleccionado, extraídos con SE, y tomando como referencia el sustantivo Zoonoseerreger:

a) Construcciones en dativo:

Rates vom 17. November 2003 zur Bekämpfung von Salmonellen und bestimmten anderen durch Lebensmittel übertragbaren Zoonoseerregern (I) die mit der Richtlinie 92/II7/EWG eingeführten spezifischen Bekämpfungsvorschriften ersetzen.

b) Construcciones en acusativo:

Änderung des Anhangs I zu berücksichtigen sind, umfassen in Bezug auf die betreffende Zoonose oder den betreffenden Zoonoseerreger i) die Häufigkeit des Auftretens in der Human- und Tierpopulation sowie in Lebensmitteln und Futtermitteln, ii) die (...)

c) Construcciones en genitivo:

(...) einschließlich in Lebens- und Futtermitteln, wenn dies im Fall der betreffenden Zoonose oder des betreffenden Zoonoseerregers angebracht ist;

d) Construcciones en nominativo:

"Zoonoseerreger" sind sämtliche Viren, Bakterien, Pilze, Parasiten oder sonstigen biologischen Einheiten, die Zoonosen (...)

e) Modificadores. Encontramos que los principales modificadores de Zoonoseerreger son los adjetivos übertragbar (transmisible), betreffend (afectado/a) y ander (otro/a):

Rates vom 17. November 2003 zur Bekämpfung von Salmonellen und bestimmten anderen durch Lebensmittel übertragbaren Zoonoseerregern in der durch die Verordnung (EU) Nr. 1086/20II der Kommission vom 27. Oktober $201 \mathrm{l}$ geänderten Fassung ist dahin

(...) der Stufe bzw. den Stufen der Lebensmittelkette, die hinsichtlich der betreffenden Zoonose bzw. des betreffenden Zoonoseerregers dafür am besten geeignet ist bzw. sind, d. h. a) auf der Ebene der Primärproduktion und/oder b) auf anderen Stufen (...)

(...) , dass angemessene und wirksame Maßnahmen zur Feststellung und Bekämpfung von Salmonellen und anderen Zoonoseerregern auf allen relevanten Herstellungs-, Verarbeitungs- und Vertriebsstufen (...).

f) Como principales complementos en genitivo de Zoonoseerreger encontramos los sustantivos Zoonose (zoonosis) y Antibiotikaresistenz (resistencia a los antibióticos): 
Punkte umfassen: I. Angaben allgemeiner Art: I.I. Vorkommen der betreffenden Zoonose bzw. des betreffenden Zoonoseerregers in dem Mitgliedstaat mit spezieller Bezugnahme auf die Ergebnisse (...)

Allgemeine Verpflichtungen (I) Die Mitgliedstaaten gewährleisten, dass Daten über das Auftreten von Zoonosen und Zoonoseerregern sowie diesbezüglicher Antibiotikaresistenzen nach Maßgabe dieser Richtlinie (...)

g) Como principales complementos en acusativo de Zoonoseerreger aparece el sustantivo Lebensmittel (alimentos):

Rates vom 17. November 2003 zur Bekämpfung von Salmonellen und bestimmten anderen durch Lebensmittel übertragbaren Zoonoseerregern (ABI. L 325, S. I) in der durch die Verordnung $\mathrm{Nr}$. 1086/20I I geänderten Fassung (im Folgenden: Verordnung Nr. 2160/

h) En cuanto a los verbos junto a los que Zoonoseerreger aparece en genitivo, destacamos fundamentalmente la forma verbal anbringen (causar, originar, traer):

(...) einschließlich in Lebens- und Futtermitteln, wenn dies im Fall der betreffenden Zoonose oder des betreffenden Zoonoseerregers angebracht ist; b) die Genehmigung spezifischer Bekämpfungsprogramme der Mitgliedstaaten (...)

i) Cuando Zoonoseerreger aparece en acusativo, suele hacerlo junto a la forma verbal untersuchen (investigar, analizar, estudiar):

(...) lebenden Tieren oder Bruteiern aus dem Herkunftslebensmittelunternehmen auf die in Spalte I genannten Zoonosen und Zoonoseerreger untersucht.

j) Las construcciones con conjunciones copulativas también son frecuentes, y así podemos encontrar el término Zoonoseerreger junto a otros sustantivos como Zoonose (zoonosis), Antibiotikaresistenz (resistencia a los antibióticos) - Salmonelle (salmonela):

2003/99/EG DES EUROPÄISCHEN PARLAMENTS UND DES RATES vom 17. November 2003 zur Überwachung von Zoonosen und Zoonoseerregern und zur Änderung der Entscheidung 90/ 424/EWG des Rates sowie zur Aufhebung der Richtlinie 92/I I7/EWG des Rates DAS

Mitgliedstaaten bewerten für ihr jeweiliges Hoheitsgebiet die Entwicklungstendenzen und Quellen von Zoonosen, Zoonoseerregern und Antibiotikaresistenzen.

(...), dass angemessene und wirksame Maßnahmen zur Feststellung und Bekämpfung von Salmonellen und anderen Zoonoseerregern auf allen relevanten Herstellungs-, Verarbeitungs- und Vertriebsstufen (...) 
k) Finalmente, podemos ver que Zoonoseerreger puede aparecer junto a grupos preposicionales con la preposición von, o formando estructuras gramaticales con in + sustantivo, gemäß + sustantivo, auf + sustantivo, sustantivo + von o bei + sustantivo:

werden. (2) Bei der Aufstellung eines koordinierten Überwachungsprogramms wird gezielt auf die Zoonosen und Zoonoseerreger in Tierpopulationen gemäß Anhang I der Verordnung (EG) Nr. 2160/2003 Bezug genommen.

Bruteiern aus dem Herkunftslebensmittelunternehmen untersucht. Die Herden und Bestände werden auf Zoonosen und Zoonoseerreger gemäß Anhang I Spalte I getestet (...)

(...), wenn sie allein handeln, nicht in der Lage, vergleichbare Daten als Grundlage für die Risikobewertung wichtiger Zoonoseerreger auf Gemeinschaftsebene zusammenzutragen. Diese Daten lassen sich auf Gemeinschaftsebene besser erheben.

(...) mit Informationen aus anderen Quellen sollten die erfassten Daten die Grundlage für die Risikobewertung von Zoonoseerregern bilden. (I5) Vorrang sollten die Zoonosen erhalten, die die menschliche Gesundheit am stärksten gefährden.

Stufen der Lebensmittelkette; I.8. die Maßnahmen, die die zuständigen Behörden bei Feststellung von Zoonosen oder Zoonoseerregern bei bestimmten Tieren oder Erzeugnissen insbesondere zum Schutz der öffentlichen Gesundheit ergriffen haben,

\section{Conclusiones}

Como hemos podido observar a lo largo de este estudio, para poder traducir adecuadamente un término es imprescindible conocer el concepto y el significado en las lenguas de trabajo. No obstante, no se trata de un mero trasvase de sentido o significado de una lengua a otra. Las complejas decisiones que a veces el traductor ha de tomar vienen también condicionadas por las estructuras gramaticales que contienen los términos que hemos de traducir en la LO, lo que supone el conocimiento del funcionamiento de dicha lengua, de sus estructuras, y de las colocaciones sintagmáticas y paratácticas, para, de esta forma, conformar todo el contexto en el cual dicho término puede aparecer, y trasvasarlo de la forma más adecuada a la LM.

Para ello, el traductor debe saber manejar no sólo herramientas lexicográficas y diccionarios especializados, en el caso de que existan para la combinación lingüística y el ámbito dentro del cual se inserta el texto que traduce, sino también ha de conocer aquellas herramientas que posibilitan el estudio de las colocaciones de las unidades de significado especializadas. Así hemos pretendido 
ilustrar esta hipótesis de investigación, mediante la confección de un corpus ad hoc para el estudio de términos relacionados con la seguridad alimentaria, que puede considerarse un subdominio del hiperónimo [veterinaria], y, dentro de él, hemos contemplado términos relativos a la patología causada por agentes que viven o provienen de los animales, como son las enfermedades conocidas como zoonosis. En este sentido, hemos constatado que el estudio y el análisis del término Zoonoseerreger (vector, agente causante de la zoonosis), las combinaciones con otras categorías gramaticales en la LO, así como su intervención en construcciones y grupos preposicionales o en coordinación con otras estructuras, ayudará en buena medida a encontrar el equivalente más adecuado para la traducción de los textos.

\section{Referencias bibliográficas}

Barelo, M. La competencia léxica en el Marco común europeo de referencia. [Recuperado

https://cvc.cervantes.es/ensenanza/biblioteca ele/carabela/pdf/58/58 027.p df: 27-48].

Candel-Mora, M. A.; Vargas-Sierra, C. (2013). An Analysis of Research Production in Corpus Linguistics Applied to Translation. En: Procedia - Social and Behavioral Sciences 95: 317-324.

Decisión 97/747/CE, por la que se fijan los niveles y frecuencias de muestreo previstas en la Directiva $96 / 23$ con vistas al control de determinadas sustancias y sus residuos en determinados productos animales.

Decisión $98 / 179$ por la que se fijan las normas específicas relativas a la toma de muestras oficiales para el control de determinadas sustancias y sus residuos en animales vivos y sus productos.

Decisión 2003/I8I/CE, por la que se modifica la Directiva 2002/657/CE en cuanto al establecimiento de límites mínimos de funcionamiento exigidos (MRPL) para determinados residuos en alimentos de origen animal.

Decisión 2004/25/CE, por la que se modifica la Directiva 2002/657/CE en cuanto al establecimiento de límites mínimos de funcionamiento exigidos (MRPL) para determinados residuos de origen animal.

Decisión de la Comisión 2002/657/CE, por la que se aplica la Directiva 96/23/CE en cuanto al funcionamiento de los métodos analíticos y la interpretación de resultados.

Decreto 69/2008, de 26 de febrero, por el que se establecen los procedimientos de las Autorizaciones Sanitarias y se crea el Registro Andaluz de Centros, Servicios y Establecimientos Sanitarios 69-2008, de 26 de febrero.

Decreto 79/20II, de 12 de abril, por el que se establecen normas sobre la distribución, prescripción, dispensación y utilización de medicamentos de 
uso veterinario y se crea el Registro de Establecimientos de Medicamentos Veterinarios de Andalucía.

Directiva 96/22/CE, por la que se prohíbe utilizar determinadas sustancias de efecto hormonal y tireostático y sustancias B-agonistas en la cría del ganado.

Directiva 96/23/CE relativa a las medidas de control aplicables respecto de determinadas sustancias $y$ sus residuos en los animales vivos y sus productos.

Directiva 200I/82/CE del Parlamento Europeo y del Consejo de 6 de noviembre de 2001 , por la que se establece un código comunitario sobre medicamentos veterinarios.

Directrices para una utilización prudente de los antimicrobianos en la medicina preventiva.

Jiménez, Rosa M. ${ }^{a}$ (2002). El concepto de competencia léxica en los estudios de aprendizaje de segundas lenguas. En Atlantis XXIV (2): I49-I62.

Ley I4/1986, de 25 de abril, General de Sanidad.

Ley 8/2003, de 24 de abril, de Sanidad Animal.

Ley $29 / 2006$, de 26 de julio, de garantías y uso racional de los medicamentos y productos sanitarios.

Ley 22/2007, de 18 de diciembre de Farmacia de Andalucía.

Ley $32 / 2007$, de 7 de noviembre, para el cuidado de los animales en su explotación, transporte, experimentación y sacrificio.

Ley 25/2009, de 22 de diciembre, de modificaciones de diversas leyes para su adaptación a la ley sobre el libre acceso de las actividades de servicio y su ejercicio.

Ley $10 / 2013$, de 24 de julio, por la que se incorporan al ordenamiento jurídico español las Directivas 2010/84/UE del Parlamento Europeo y del Consejo, de 15 de diciembre de 20I0, sobre farmacovigilancia, y 20II/62/UE del Parlamento Europeo y del Consejo, de 8 de junio de 2011 , sobre prevención de la entrada de medicamentos falsificados en la cadena de suministro legal, y se modifica la Ley 29/2006, de 26 de julio, de garantías y uso racional de los medicamentos y productos sanitarios.

Orden de 20 de noviembre de 2012, por la que se aprueba el listado de presentaciones, principios activos y formas farmacéuticas que entran a formar parte de los depósitos especiales en los botiquines veterinarios.

Orden de 4 de diciembre de 2012, por la que se establece el procedimiento de comunicación de prescripción excepcional de un medicamento veterinario autorizado en otro Estado Miembro de la Unión Europea.

Orden PRE/2833/2009, de 19 de octubre, por la que se modifica el anexo I del Real Decreto 1246/2008, de 18 de julio, por el que se regula el procedimiento de autorización, registro y farmacovigilancia de los medicamentos veterinarios fabricados industrialmente. 
Orden PRE/24/36/20I3, de 26 de diciembre, por la que se modifican los anexos I, II, y IV del Real Decreto 1675/20II2, de I4 de diciembre, por el que se regulan las recetas oficiales y los requisitos especiales de prescripción y dispensación de estupefacientes para uso humano y veterinario.

Real Decreto 1945/1983, de 22 de junio, por el que se regulan las infracciones y sanciones en materia de defensa del consumidor y de la producción agroalimentaria.

Real Decreto 109/1995, de 27 de enero, sobre medicamentos veterinarios.

Real Decreto 1749/1998, por el que se establecen las medidas de control aplicables a determinadas sustancias y sus residuos en los animales vivos y sus productos.

Real Decreto 2178/2004, por el que se prohíbe utilizar determinadas sustancias de efecto hormonal y tireostático y $\mathrm{B}$-agonistas de uso en la cría del ganado.

Real Decreto 640/2006, de 26 de mayo, por el que se regulan determinadas condiciones de aplicación de las disposiciones comunitarias en materia de higiene, de la producción y comercialización de los productos alimenticios.

Real Decreto 1246/2008, de 18 de julio, por el que se regula el procedimiento de autorización, registro y farmacovigilancia de los medicamentos veterinarios fabricados industrialmente.

Real Decreto $361 / 2009$, de 20 de marzo, por el que se regula la información sobre la cadena alimentaria que debe acompañar a los animales destinados a sacrificio.

Real Decreto 488/2010, de 23 de abril, por el que se regulan los productos zoosanitarios.

Real Decreto 824/2010, de 25 de junio, por el que se regulan los laboratorios farmacéuticos, los fabricantes de principios activos de uso farmacéutico y el comercio exterior de medicamentos y medicamentos en investigación.

Real Decreto II32/2010, de 10 de septiembre, por el que se modifica el Real Decreto 109/1995, de 27 de enero, sobre medicamentos veterinarios.

Real Decreto I275/20I I, de I6 de septiembre, por el que se crea la Agencia estatal "Agencia Española de Medicamentos y Productos Sanitarios" y se aprueba su Estatuto.

Real Decreto 1675/2012, de 14 de diciembre, por el que se regulan las recetas oficiales y los requisitos especiales de prescripción y dispensación de estupefacientes para uso humano y veterinario.

Real Decreto 544/2016, de 25 de noviembre, por el que se regula la venta a distancia al público de medicamentos veterinarios no sujetos a prescripción veterinaria.

Real Decreto Legislativo I/2015, de 24 de julio, por el que se aprueba el texto refundido de la Ley de garantías y uso racional de los medicamentos y productos sanitarios. 
Recomendación de la Comisión de 14 de enero de 201 I, por la que se establecen directrices para la distinción entre materias primas para piensos, aditivos para piensos, biocidas y medicamentos veterinarios.

Reglamento (CE) n 1950/2006 de la Comisión, de 13 de diciembre de 2006, que establece una lista de sustancias esenciales para el tratamiento de équidos, de conformidad con la Directiva 200 I/82/CE del Parlamento Europeo y del Consejo por la que se establece un código comunitario sobre medicamentos veterinarios.

Reglamento (CE) n 470/2009 del Parlamento Europeo y del Consejo, de 6 de mayo de 2009, por el que se establecen procedimientos comunitarios de fijación de límites de residuos de las sustancias farmacológicamente activas en los alimentos de origen animal, se deroga el Reglamento (CE) $n^{\circ}$ 2377/90 del Consejo y se modifica la Directiva 200 I/82/CE del Parlamento Europeo y del Consejo y el Reglamento (CE) n 726/2004 del Parlamento Europeo y del Consejo.

Reglamento (CE) n 37/2010 de la Comisión, de 22 de diciembre de 2009, relativo a las sustancias farmacológicamente activas y su clasificación por lo que se refiere a los límites máximos de residuos en los productos alimenticios de origen animal.

Resolución de 10 de febrero de 2010, de la Secretaría General Técnica, por la que se publica el Convenio de Colaboración de la Consejería de Agricultura y Pesca de la Junta de Andalucía y el Consejo Andaluz de Colegios Oficiales de Veterinarios para la edición, distribución y gestión de la numeración de las recetas veterinarias en Andalucía.

Resolución de 10 de junio de 2013, de la Dirección General de la Producción Agrícola y Ganadera, por la que se da publicidad a los modelos normalizados de receta veterinaria.

Sánchez, M. M. (2017). Metodología de corpus y formación en la traducción especializada (inglés-español): una propuesta para la mejora de la adquisición de vocabulario especializado. En Revista de Lingüística y Lenguas Aplicadas I2: I37-I50.

(20l3). El léxico en el aula de traducción: diseño de un modelo de adquisición de la competencia léxica traductora (inglés-español). En: Tonos Digital. Revista de Estudios Filológicos 24. [Recuperado de: https://www.um.es/tonosdigital/znum24/secciones/tritonos-2competencia_lexica_traductora.htm]. 